\title{
Construcción de espacios muestrales asociados a distintos tipos de sucesos: un estudio exploratorio con estudiantes de Educación Primaria
}

\section{Building sample spaces linked to different events: an exploratory study with primary school students}

\section{Luis Armando Hernández-Solís, ${ }^{1}$ Carmen Batanero, ${ }^{2}$ María M. Gea, ${ }^{3}$ Rocío Álvarez-Arroyo ${ }^{4}$}

\begin{abstract}
Resumen: Se presenta un estudio exploratorio en que se investiga la construcción del espacio muestral asociado a varios tipos de sucesos. Se analizan las respuestas de 55 estudiantes costarricenses de $6^{\circ}$ curso de educación primaria (entre 11 y 12 años), 29 de una escuela privada y 26 de otra pública. Mediante dos ítems de elaboración propia, uno en contexto de urnas y otro de ruletas, se les pide construir el espacio muestral compatible con la descripción de un suceso seguro, probable, equiprobable e imposible. Se categorizan las respuestas atendiendo al tipo de suceso que tendría lugar, teniendo en cuenta los espacios muestrales que construyen, y analizando las diferencias en los dos contextos y en las dos escuelas. Las tareas más sencillas fueron construir un espacio muestral compatible con un suceso posible y equiprobable, mientras que pocos estudiantes construyen correctamente un espacio muestral que corresponda al suceso seguro e imposible. Estos resultados apoyan otras investigaciones previas que indican la dificultad que para los estudiantes tienen los conceptos de seguro e imposible.
\end{abstract}

\footnotetext{
Fecha de recepción: 09 de junio de 2020. Fecha de aceptación: 02 de noviembre de 2020

1 Universidad Estatal a Distancia-Costa Rica, Ihernandez@uned.ac.cr, orcid.org/0000-0003-2956-8102

2 Universidad de Granada, batanero@ugr.es, orcid.org/0000-0002-4189-7139

3 Universidad de Granada, mmgea@ugr.es, orcid.org/0000-0002-5229-0121

${ }_{4}^{4}$ Universidad de Granada, rocioaarroyo@ugr.es, orcid.org/0000-0002-3201-8542
} 
Palabras clave: tipos de sucesos, intuiciones, espacio muestral, alumnos de educación primaria.

\begin{abstract}
We present an exploratory study in which we analyse the construction of the sample space associated with various types of events. We analyse the responses of 55 students from $6^{\text {th }}$ degree of primary education, 29 of them from a private school and 26 from a public school. We propose them two items one in the urn context and another in roulettes context, in which the students are asked to build the sample space compatible with the description of a certain, probable, equiprobable and impossible event. The responses are categorized according to the type of event that would take place, when taking into account the sample spaces the children construct, and we analyse the differences in the two contexts and schools. The simpler tasks were building a sample space compatible with a possible and equiprobable event, while few students correctly constructed a sample space corresponding to the certain and impossible event. These results support other previous research that indicates the difficulty for students of the concepts of certain and impossible.
\end{abstract}

Keywords: types of events, intuition, sample space, primary school students.

\title{
INTRODUCCIÓN
}

Numerosos autores, entre otros Fischbein (1975) y Fischbein y Gazit (1984), resaltaron el papel de la enseñanza para educar las intuiciones en probabilidad, que con frecuencia se desarrollan inadecuadamente sin una instrucción específica, y que son necesarias para tomar decisiones correctas en la vida cotidiana y profesional.

En estas situaciones es con frecuencia posible medir la incertidumbre de un cierto fenómeno por medio de la probabilidad, que es un instrumento para expresar, cuantificar y modelizar esta incertidumbre (Pratt y Kazak, 2018). Consecuentemente, una finalidad principal de la enseñanza de la probabilidad es proporcionar al niño experiencia con las situaciones aleatorias que le permitan manejarse en un mundo incierto, donde estos fenómenos están presentes en las actividades más variadas (Jones, Langrall y Money, 2007). Esta necesidad ha repercutido en el currículo de matemáticas, donde se ha incorporado, en las 
últimas décadas, la probabilidad en la educación primaria (Australian Curriculum, Assessment and Reporting Authority, 2013; Common Core State Standards Initiative, 2010, Ministerio de Educación, Cultura y Deporte, España, 2014; National Council of Teachers of Mathematics, 2000). Además, la probabilidad se incluye en el currículo de matemáticas por ser una rama de esta ciencia que enriquece las restantes e interacciona con ella (Franklin et al. 2007).

En Costa Rica se aprobaron nuevos programas escolares de Matemática en 2012, en donde el área de Estadística y Probabilidad adquirió un relieve mucho mayor, respecto a los anteriores currículos nacionales, que incluían contenidos de probabilidad de forma muy reducida en educación primaria. Ello incidía en la poca trascendencia que se les daba a estos temas que, además, no tenían continuidad en educación secundaria. El aumento de más tópicos de probabilidad en los nuevos programas escolares de Matemática del Ministerio de Educación Pública (MEP, 2012) tienen como propósito “...formar en el pensamiento aleatorio y en el desarrollo de capacidades para abordar el azar, lo impredecible, la incertidumbre, características que participan en el conocimiento y en la vida de múltiples maneras" (p.55). Se justifica este énfasis señalando "El lugar relevante que se da a esta área obedece al papel que juega la información y el manejo del azar en la sociedad moderna" (p. 55).

Vásquez y Alsina $(2015 ; 2017)$ sugieren que esta incorporación plantea un desafío, pues muchos profesores actuales de educación primaria no han recibido suficiente formación en probabilidad. En su trabajo indican que algunos necesitan mejor preparación en nociones básicas de probabilidad -tales como experimento aleatorio, espacio muestral, suceso probable o seguro-. Esta carencia ha sido también evidenciada en Costa Rica (Alpízar et al., 2012; Alpízar, Chavarría y Oviedo, 2015). Debido a la poca relevancia de estos contenidos en planes de estudio anteriores, tampoco hay investigaciones sobre el aprendizaje de la probabilidad a nivel escolar en Costa Rica y las realizadas en otros contextos son escasas.

Con el fin de orientar al profesor en el tipo de tareas adecuadas a los estudiantes que cursan la educación primaria, hemos iniciado un proyecto de investigación dirigido a conocer el razonamiento probabilístico típico de estos estudiantes. En concreto, en este trabajo nos centramos en estudiantes de $6^{\circ}$ curso de educación primaria (edades entre 11 y 13 años), eligiendo este curso porque con él se concluye esta etapa educativa. Además, el grupo de estudiantes que forma la muestra han seguido el currículo actual de Matemática (MEP, 2012), que a partir de 2015 se aplicó de forma completa en todos los niveles 
educativos. En concreto, los contenidos de probabilidad en la educación primaria que han seguido los estudiantes de la muestra son:

Primer ciclo ( $1^{\circ}$ a $3^{\circ}$ cursos): Identificar situaciones aleatorias y seguras dentro de la cotidianidad y eventos asociados con ellas. Clasificar eventos aleatorios en más o menos probables para situaciones o experimentos particulares. Identificar eventos de acuerdo con los resultados simples que están vinculados con ellos (p. 147).

Segundo ciclo ( $4^{\circ}$ a $6^{\circ}$ cursos): Identificar eventos más probables, menos probables o igualmente probables de acuerdo con el número de resultados simples pertenecientes a cada evento. Determinar probabilidades elementales vinculadas con eventos particulares. Plantear y resolver problemas vinculados con situaciones aleatorias (p. 247).

Puesto que las investigaciones anteriores sobre el razonamiento probabilístico de los estudiantes de esta edad se han realizado cuando ellos no habían recibido enseñanza de la probabilidad, nuestra investigación aporta información original, que puede ser comparada con los resultados de dichos estudios. Nuestro trabajo, además, se enfoca, tanto en el lenguaje del azar, cuyo conocimiento es considerado por Watson y Moritz (2013) una parte básica de la cultura estadística, como en la interpretación que los estudiantes realizan del espacio muestral en un experimento aleatorio sencillo. Específicamente evaluamos su conocimiento de los términos suceso seguro, imposible, posible y equiprobable, cada uno de los cuales corresponde a un concepto; además, exploramos la forma en que los estudiantes relacionan estas nociones con el conjunto de resultados de un experimento aleatorio simple (que matemáticamente sería el espacio muestral).

Para evaluar el conocimiento de los términos mencionados en las investigaciones previas a la nuestra, se pide al niño escribir o identificar una frase con cada una de las palabras posible, imposible, seguro o indicar un sinónimo de las mismas. En nuestro trabajo se pedirá construir un espacio muestral con un número pequeño de sucesos, de modo que un cierto suceso descrito en el enunciado de la tarea corresponda a un suceso seguro, posible, equiprobable o imposible en dicho experimento. Se comparan, además, los espacios muestrales construidos por los estudiantes en el contexto de ruletas y de urnas; así como los resultados respecto al tipo de institución educativa, privada o pública. Nos interesamos por el espacio muestral, pues como Keren (1984) indica, los estudiantes construyen espacios muestrales informales, que no son siempre los esperados por el investigador y que es necesario conocer qué espacios muestrales construyen para comprender sus estrategias en la resolución de problemas de probabilidad. 


\section{FUNDAMENTOS}

\section{INTUICIÓN Y LENGUAJE DE LA PROBABILIDAD}

Puesto que la enseñanza de la probabilidad en la educación primaria es básicamente intuitiva y depende sustancialmente del lenguaje que los estudiantes dominan, dos puntos a tener en cuenta en nuestro trabajo son las intuiciones que llevan al tema y el lenguaje que se utiliza en la clase de probabilidad.

Respecto a las intuiciones, Fischbein (1987) las considera parte de conducta inteligente. Las describe como adquisiciones cognitivas que intervienen en las acciones cotidianas y guían el razonamiento. El autor diferencia entre intuiciones primarias (que se adquieren sin enseñanza, a partir de la experiencia) y secundarias (que son consecuencia de la instrucción); cada uno de estos tipos de intuiciones pueden, a su vez, ser correcta o incorrecta. Fischbein (1975) indica que algunas personas adquieren intuiciones incorrectas en probabilidad que son muy difíciles de cambiar a partir de una cierta edad, por lo que es conveniente identificarlas a tiempo, para darles ocasión de corregirlas. En nuestro trabajo analizamos la intuición que el niño tiene del espacio muestral, es decir, del conjunto de resultados posibles de un experimento aleatorio sencillo y cómo relaciona este espacio muestral con un suceso seguro, posible, equiprobable o posible. Cabe señalar que, aunque los estudiantes han recibido instrucción en probabilidad, el tipo de tareas que se les presentan en los ítems 1 y 2 no forman parte de la enseñanza recibida, de acuerdo con la información proporcionada por los docentes a cargo de los grupos y la revisión de los libros de texto utilizados.

Puesto que los estudiantes de la muestra están recibiendo instrucción en probabilidad, es importante considerar el lenguaje que ellos utilizan, pues en la educación primaria los conceptos matemáticos se introducen mostrando ejemplos, a partir del lenguaje propio de la edad y contexto. Poco a poco, se dota a estos ejemplos y a las definiciones informales construidas por los estudiantes de mayor abstracción (Schleppegrell, 2007). El lenguaje tiene también una gran importancia, tanto para la comunicación de ideas matemáticas, como para la resolución de problemas, ya que los objetos matemáticos no son ostensivos y necesitan ser representados mediante diferente lenguaje (Godino, Batanero y Font, 2007). Por otro lado, Vygotsky (2012) considera que el lenguaje está unido al pensamiento y que su adquisición es una parte central en el aprendizaje, que es para el autor, un proceso social. 
Hernández (2015) resalta la importancia de conocer el vocabulario que los alumnos asocian a las situaciones aleatorias y a la probabilidad, ya que algunos términos que utilizamos en matemáticas no tienen el mismo significado en su uso coloquial fuera del aula. Entre los términos asociados a la probabilidad que tienen esta diferencia se sitúan los investigados en este trabajo, ya que cuando se usa en una frase la palabra "seguro" no siempre se quiere decir que el hecho descrito se producirá, mientras que en probabilidad el suceso seguro incluye todos los sucesos elementales del espacio muestral y, por tanto, ocurre siempre.

Igualmente, en algunas investigaciones que han analizado el lenguaje probabilístico en los libros de texto, por ejemplo, en Gómez, Ortiz, Batanero y Contreras (2013) y Ortiz, Albanese y Serrano (2016), se sugiere que el lenguaje verbal se usa para sugerir un significado específico de la probabilidad. Por ejemplo, los términos caso favorable o juego equitativo están ligados al significado clásico, mientras que frecuencia y repetición están asociados al frecuencial. También se reitera que hay palabras del lenguaje ordinario, entre ellas las usadas en nuestro trabajo, que no tienen un sentido equivalente en matemáticas.

\section{ANTECEDENTES DEL TRABAJO}

Las investigaciones sobre el lenguaje de probabilidad se han llevado a cabo en un periodo en que la probabilidad no se enseñaba en la escuela primaria y se han realizado en muchos casos con estudiantes mayores que los que forman parte de nuestro estudio. Por ejemplo, Green (1983), en un estudio con 3000 estudiantes ingleses de 11 a 16 años, les propuso un cuestionario que recoge versiones en papel y lápiz de muchos de los experimentos realizados con dispositivos físicos por Piaget e Inhelder (1951). Uno de los apartados de su cuestionario evaluaba el conocimiento del lenguaje de la probabilidad por parte de los estudiantes. Concretamente, analizaron varios términos, entre ellos algunos equivalentes a los usados en nuestro trabajo, específicamente "no puede ocurrir" (para nosotros imposible), "probable", "improbable", "posible", "cincuenta por ciento de probabilidades" o "igual posibilidad" (que sería equivalente a equiprobable), "siempre ocurre" (para nosotros seguro). Las tareas propuestas fueron pedir al niño explicar con sus propias palabras qué significa el término, dar sinónimos de las palabras o escribir una frase utilizándola. El autor obtuvo en los estudiantes de $6^{\circ}$ curso $49 \%$ de respuestas correctas para el significado de "no puede ocurrir", $22 \%$ en "siempre ocurre", 84\% en "posible" y $18 \%$ en "igual posibilidad". 
La investigación de Green (1983) fue replicada por Cañizares (1997) con 320 estudiantes españoles de 10 a 14 años, y la autora obtuvo en los estudiantes de $6^{\circ}$ curso $46,2 \%$ de respuestas correctas para el significado de "no puede ocurrir", 23\% en "siempre ocurre", 81\% en "posible", 43\% en "igual posibilidad". Sus resultados fueron mejores que los del estudio de Green (1983), encontrándose en ambos casos la mayor dificultad en comprender la idea de suceso seguro (siempre ocurre) e igual posibilidad (equiprobable).

Nacarato y Grando (2014) realizaron entrevistas a 12 estudiantes de $6^{\circ}$ curso de Educación Primaria en Sao Paulo, Brasil, a los que pidieron ordenar en una escala de mayor a menor probabilidad los términos improbable, poco probable, probable y muy probable. Esta experiencia fue sugerida anteriormente por Ernest (1984), quien también añadió que se podría pedir a los estudiantes asignar a cada una de estas palabras un número o fracción entre 0 y 1 . Nacarato y Grando indicaron que ninguno de los estudiantes ordenó los términos en la forma esperada por los investigadores, siendo lo más frecuente cambiar el orden de probable y muy probable.

Watson (2005) resalta el hecho de que algunos estudiantes interpretan la probabilidad del 50\% como que cualquier resultado puede ocurrir, en vez de asociarla a un experimento con dos sucesos equiprobables. En vez de ello, lo ligan con un experimento con dos resultados, aunque no sean equiprobables e incluso a experimentos con más de dos resultados, por ejemplo, lanzar un dado.

Watson y Moritz (2010), por su parte, analizaron los resultados a dos preguntas de una encuesta realizada con 2700 estudiantes australianos de 5 a 11 años, donde se pedía evaluar frases tomadas de las noticias en los medios de comunicación, utilizando la escala cualitativa de probabilidad, es decir, utilizando palabras que expresasen la probabilidad del suceso, como "probable", "imposible", "muy o poco probable" y colocándolas en la recta numérica entre 0 y 1. La mayoría de los estudiantes situó el término imposible al comienzo de la escala de probabilidad, pero no siempre en el extremo, e igualmente no siempre se interpretó seguro como algo que siempre ocurre. Se observó también que al avanzar la edad mejoraba la ordenación de los términos dados por parte de los estudiantes.

Hernández-Salmerón, López-Martín y Batanero (2017) realizan una investigación con 89 estudiantes de $1^{\circ}$ y $2^{\circ}$ curso de educación secundaria obligatoria en España (13-14 años), utilizando un cuestionario sobre el conocimiento del lenguaje del azar. Una de las tareas fue escribir sinónimos de las palabras "imposible", "posible", "igual posibilidad", "poca posibilidad", "seguro", "muy posible". El porcentaje de estudiantes que no pudo dar ningún sinónimo correcto para imposible fue 
de $48,2 \%$ en $1^{\circ}$ curso y de $33 \%$ en el $2^{\circ}$ curso, mientras que estos porcentajes crecieron al $73,2 \%$ y $60,6 \%$ para seguro. Las autoras indican que algunos alumnos interpretaron como seguro frases como "puede que sí o puede que no ocurra" e indica la necesidad de dedicar algún tiempo a discutir con los estudiantes el significado que en matemática se da a los términos relacionados con el azar.

En todas estas investigaciones se pregunta a los estudiantes por sinónimos de los términos probabilísticos, se les pide ordenarlos en una escala de probabilidad, o bien se les piden describan sucesos que sean probables, imposibles, seguros, etc. Nosotros tomaremos una aproximación diferente, pues les presentamos una tarea, con el dibujo de un dispositivo aleatorio (ruletas o urnas) cuya composición deben fijar para que un determinado suceso tenga una cierta probabilidad, que se les precisa mediante una expresión del lenguaje verbal probabilístico.

También tendremos en cuenta las investigaciones sobre la comprensión del espacio muestral que es, de acuerdo a Langrall y Mooney (2005), fundamental para el razonamiento probabilístico. Aunque Piaget e Inhelder (1951) sugieren que los estudiantes de 7 años pueden listar todos los resultados de un experimento aleatorio sencillo, Borovcnik y Bentz (1991) encontraron algunos estudiantes de 11 años que no llegaban a completar esta tarea. La comprensión del espacio muestral según Horvath y Lehrer (1998) requiere coordinar las siguientes capacidades: a) reconocer todos los posibles resultados de un experimento, b) poderlas describir en forma completa y c) relacionar el espacio muestral con la mayor o menor posibilidad de cada resultado del experimento. Los autores indican que algunos estudiantes no llegan a comprender este último punto.

Por otro lado, Langrall y Money (2005) proponen una escala de valoración de la comprensión de los estudiantes del espacio muestral, pidiéndoles que completen el espacio muestral de un experimento; por ejemplo, completar todas las posibilidades que pueden ocurrir al lanzar un dado. El nivel más bajo, donde se sitúan muchos estudiantes, consiste en concentrarse simplemente en un suceso, por ejemplo, para construir el espacio muestral en un experimento consistente en extraer fichas de una urna, considerar solo fichas del mismo color. Ello se debe a que su razonamiento es determinista y no tienen en cuenta todos los sucesos que podrían ocurrir en un experimento. El segundo nivel se alcanza cuando se es capaz de listar varios sucesos diferentes del espacio muestral, pero no todos; el tercero si se consigue describir exhaustivamente todos los sucesos posibles de un espacio muestral y el más elevado ser capaz de realizar una lista de los elementos del espacio muestral de un experimento compuesto sencillo, por ejemplo, lanzar dos monedas al aire. 
La clasificación de los casos como favorables o desfavorables para que ocurra un cierto suceso, es un paso crucial para determinar la probabilidad del mismo. Sin embargo, Francisco y Maher (2005) y Nilsson (2007) muestran que es una tarea compleja para los estudiantes y que algunos que pueden listar todos los sucesos de un espacio muestral tienen dificultad en determinar el numerador y denominador para aplicar la regla de Laplace, porque no separan bien los casos en favorables y desfavorables.

\section{MÉTODO}

La muestra estuvo formada por 55 estudiantes (27 mujeres y 28 hombres) de $6^{\circ}$ curso de educación primaria, 40 de 11 años y 15 de 12 años, de los cuales 29 realizaban sus estudios en una institución privada y 26 en una institución pública (estatal-gratuita), ambas de la provincia de Cartago, Costa Rica. Las instituciones son muy cercanas geográficamente, a menos de dos kilómetros de distancia; y tienen aproximadamente la misma cantidad de estudiantes en educación primaria (rondan los 450 estudiantes). Los estudiantes de la escuela privada provienen de diferentes cantones y distritos de la provincia, a diferencia de la escuela pública, donde más del 90\% de los estudiantes de la muestra pertenecen al distrito donde está ubicada la escuela. Aunque no se hizo un estudio económico, nuestro conocimiento de las instituciones educativas indica que existen marcadas diferencias en este aspecto entre los estudiantes de una y otra escuela.

Ambas instituciones se rigen por el programa de estudios de Matemática (MEP, 2012). Sin embargo, mientras que en la escuela pública se imparten ocho lecciones semanales de Matemática, según lo que establece el Ministerio de Educación Pública de Costa Rica; en la escuela privada se imparten solo cinco lecciones semanales de Matemática, una de ellas dedicada específicamente al área de Estadística y Probabilidad, desde el 2016. Las docentes encargadas de los cursos nos informaron que ambos grupos desarrollaron los contenidos sugeridos en las orientaciones curriculares descritas en la introducción de este trabajo a partir del mismo libro de texto, dándole gran relevancia a este recurso y a las actividades "lápiz y papel".

A dicha muestra se aplicó un cuestionario con diferentes preguntas sobre probabilidad. En este trabajo se analizan los dos ítems presentados en la figura 1, que son de creación propia y tratan de la construcción de un espacio 
muestral y de los conceptos de suceso seguro, imposible, posible y equiprobable en un contexto de juego de azar. Otros ítems relacionados con la comparación de probabilidades en contexto de urnas de han publicado en Hernández-Solís, Batanero, Gea, M. y Álvarez-Arroyo (2021).

Para resolver la tarea, el estudiante ha de pensar en un experimento aleatorio que se pueda realizar con el dispositivo dado (ruleta o urna) y donde el suceso pedido tenga la probabilidad expresada verbalmente. Para ello, debe imaginar un conjunto dicotómico de posibilidades divididas en dos subconjuntos (casos favorables y casos desfavorables).

Ítem 1: María y Esteban juegan con una ruleta. María gana un confite si la aguja que gira cae en el 1 y Esteban gana un confite si cae en el 2. Coloca en las siguientes ruletas los números que consideres oportunos para que se cumpla:

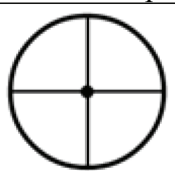

(A) María gana seguro

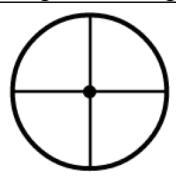

(B) Es posible que María gane

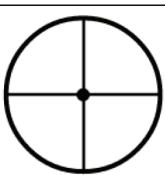

(C) María y Esteban tienen igual posibilidad de ganar

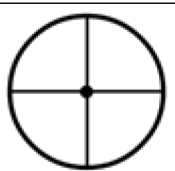

(D) Es imposible que María gane

Ítem 2. María gana cuando saca ficha negra. Pinta en las siguientes urnas tantas fichas negras y blancas, como consideres oportuno, para que ocurra:

\begin{tabular}{|c|c|c|c|}
\hline $\mid$ & $\mid$ & $\mid$ & $\mid$ \\
$\begin{array}{c}\text { (A) María gana } \\
\text { seguro }\end{array}$ & $\begin{array}{c}\text { (B) Es posible que } \\
\text { María gane }\end{array}$ & $\begin{array}{c}\text { (C) María tienen } \\
\text { igual posibilidad de } \\
\text { ganar que de perder }\end{array}$ & $\begin{array}{c}\text { (D) Es imposible } \\
\text { que María gane }\end{array}$ \\
\hline
\end{tabular}

Figura 1. Ítems analizados en este trabajo.

De acuerdo a la figura 1, se utilizarán los contextos de ruletas, divididas en cuatro partes iguales y fichas de dos colores en urnas. Según Cañizares (1997), el contexto de la ruleta favorece las comparaciones de tipo parte-todo, mientras el contexto de fichas utilizado en el segundo ítem, donde se puede aplicar la regla de Laplace, favorece la comparación entre casos favorables y casos desfavorables (comparación parte-parte). Cada uno de los ítems presenta cuatro apartados; en el primero se pide generar un espacio muestral donde el suceso "María gana" sea seguro, en el segundo que sea posible, en el tercero que sea un suceso equiprobable a su contrario y el cuarto que sea un suceso imposible. 
En el primero de los ítems el juego se realiza utilizando la representación de una ruleta donde, si los espacios de división fuesen de diferente amplitud, no se podría aplicar la regla de Laplace, sino la probabilidad geométrica para calcular probabilidades, y esto excede lo establecido para este nivel educativo, por lo que se ha optado en dividir cada ruleta en cuatro sectores circulares con igual área. El estudiante puede crear un espacio muestral de hasta cuatro sucesos diferentes (cuatro números distintos), en cuyo caso todos los sucesos elementales serían equiprobables, e igualmente la probabilidad de ganar el juego de los niños imaginarios del enunciado. Pero si alguno de los números se repite (por ejemplo, construir una ruleta numerada $1,1,2,3$ ), se consigue un espacio muestral donde el suceso "1" tiene doble probabilidad que los restantes, y por tanto María doble probabilidad de ganar que Esteban. Para conseguir el suceso seguro se debe repetir cuatro veces el número 1 ("María gana seguro") y para lograr el imposible ("Es imposible que María gane"), se debe excluir el número 1 de todos los sectores. En resumen, la resolución de la tarea requiere la intuición o el conocimiento del experimento aleatorio, y suceso, sucesos posibles del experimento, suceso favorable (gana María) y desfavorable (no gana María), así como de la intuición de seguro, posible, equiprobable e imposible, según el apartado.

En el segundo ítem (contexto de urnas), la única restricción es que el espacio muestral consta de fichas negras y blancas, y María gana sacando la ficha negra. Por ello se puede construir un espacio muestral con cualquier número de fichas, aunque es de esperar que el estudiante piense en un número pequeño de ellas. De nuevo ha de pensar en el experimento aleatorio y sus posibles resultados, así como en los casos favorables, casos desfavorables y los sucesos del tipo dado en el apartado. Para lograr el suceso seguro, debe rellenar la urna sólo con fichas negras y para el imposible sólo con fichas blancas, en cualquier cantidad para los dos tipos de suceso. El suceso equiprobable constará del mismo número de fichas blancas y de fichas negras, y el posible cualquier composición con al menos una ficha de cada color.

Los cuestionarios se aplicaron a los estudiantes durante una de sus clases de matemáticas, con asistencia del profesor y de uno de los investigadores, quien explicó a los estudiantes lo que se les pedía en la tarea. Los estudiantes las completaron con interés e individualmente por escrito, y la actividad sirvió para la clase de probabilidad, resultando de interés para los estudiantes y el profesor. En el momento de la aplicación del cuestionario, los niños estaban comenzando el curso lectivo, por lo que no habían estudiado contenidos de probabilidad correspondientes al $6^{\circ}$ curso. 
Recogidos los datos, se analizaron los cuestionarios y se clasificaron los tipos de respuesta en cada apartado, de acuerdo al tipo de espacio muestral construido.

\section{RESULTADOS}

A continuación, se describen algunas posibles respuestas correctas e incorrectas para cada uno de los apartados de los ítems, presentando algunos ejemplos de respuestas de los estudiantes que participaron en el estudio.

Espacio muestral que corresponde al suceso seguro: La respuesta al primer apartado de cada ítem sería correcta si se construye un espacio muestral formado sólo por casos favorables al suceso gana María, lo que asegura que María gane el juego. En la figura 2, presentamos dos ejemplos, uno para cada ítem. En el primero de ellos, el niño numera todos los sectores de la ruleta con el número uno y en el segundo llena la urna con cuatro fichas negras. El primer ejemplo es la única posibilidad correcta para que el suceso "gana María" sea seguro en el primer ítem, mientras en el segundo caso se podría haber utilizado cualquier cantidad de fichas negras.

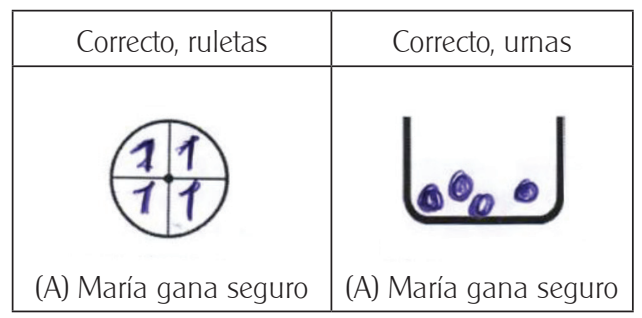

Figura 2. Espacios muestrales correctos construidos para un suceso seguro.

En los ejemplos incorrectos que se muestran en la figura 3, se propone un espacio muestral con diferentes tipos de sucesos, incluyendo, en consecuencia, casos favorables y casos desfavorables al suceso "gana María". Por tanto, no puede asegurarse que María ganará.

En el ejemplo incorrecto 1 presentado para el contexto de la ruleta, la probabilidad de ganar María sería 1/4, igual que la de su compañero, quien también tiene un caso favorable entre cuatro y por tanto no es seguro que María gane el juego, sino que se ha construido el espacio muestral que corresponde a un 
suceso equiprobable (tanto María como Esteban tienen igual probabilidad de ganar). En el ejemplo incorrecto 2, el estudiante construye un espacio muestral para el suceso "muy probable que María gane", ya que aparecen tres casos favorables de cuatro posibles, por lo que la probabilidad de que María gane es $3 / 4$. En el ejemplo incorrecto 3, se incluyen cinco casos favorables frente a dos desfavorables, y en el ejemplo 4, se presentan siete casos favorables y uno desfavorable. Hay que señalar, que en los ejemplos incorrectos del 2 al 4 los estudiantes confunden muy probable con seguro, confusión que ya apareció en las investigaciones de Cañizares (1997), Green (1983) y Hernández-Salmerón et al. (2017). Nuestra explicación alternativa para estos razonamientos es un sentido de justicia, unido a la intuición que se aproxima al concepto de suceso seguro, ya que se da a María una alta probabilidad de ganar; sin embargo, se dejan la posibilidad de que gane Esteban, pues quizás no sería natural para el niño dejarlo sin oportunidades de ganar, ya que participa en el juego.

\begin{tabular}{|l|l|l|l|}
\hline Incorrecto 1, ruletas & Incorrecto 2, ruletas & Incorrecto 3, urnas & Incorrecto 4, urnas \\
\hline
\end{tabular}

Figura 3. Espacios muestrales incorrectos construidos para un suceso seguro.

Espacio muestral que corresponde a un suceso posible. En el segundo apartado de cada ítem se pide construir un espacio muestral en el que María tiene posibilidad, pero no seguridad, de ganar el juego. Por tanto, se admite una amplia variedad de soluciones correctas, pues excluido el caso de imposibilidad y el de seguridad, consideraríamos correctas todas las demás respuestas.

En esta tarea no se encontraron espacios muestrales incorrectos para el suceso posible, aunque hubo graduación en el sentido que algunos estudiantes produjeron espacios muestrales que proporcionaban sucesos muy poco probables, probables, equiprobables o muy probable, pero no seguros ni imposibles. Mostramos en la figura 4 un par de ejemplos que corresponden a espacios muestrales correctos, ya que se incluyen tanto casos favorables como desfavorables. De hecho, son bastantes los que producen sucesos equiprobables, esto 
se ilustra con el ejemplo de urnas mostrado en la figura 4. De nuevo, conjeturamos que el sentido de justicia de los estudiantes los lleva a construir un espacio muestral en que los dos jugadores tengan la misma probabilidad de ganar.

\begin{tabular}{|r|c|}
\hline Correcto, ruletas & Correcto, urnas \\
\hline & \begin{tabular}{|l|l|}
\hline 2 & 7 \\
\hline 1 & 1
\end{tabular} \\
\hline (B) Es posible que María gane & (B) Es posible que María gane \\
\hline
\end{tabular}

Figura 4. Espacios muestrales construidos para un suceso posible.

Espacio muestral que corresponde al suceso equiprobable. El tercer apartado requiere que los estudiantes construyan un espacio muestral en que María y Esteban tengan las mismas posibilidades de ganar. Los estudiantes parecen tener clara la idea de equiprobabilidad, pues construyen en general ejemplos correctos de espacios muestrales con el mismo número de casos favorables y casos desfavorables, como se observa en la figura 5.

En lo ejemplos correctos 1, 2 y 4, los estudiantes usan dos casos favorables para cada jugador, con la diferencia de disposición entre ruletas en el 1 y 2, donde en el primero los casos favorables se alternan y en el segundo se encuentran consecutivos. Aunque este aspecto pueda parecer no relevante, algunos estudiantes asocian mayor o menor probabilidad según la disposición u orden de los sectores en una ruleta o dispositivo continuo (Maury, 1984). En el ejemplo 3, se da una composición en donde el niño considera otros casos desfavorables además del "2" con el que juega Esteban. En el ejemplo 5 de urnas, cabe destacar la cantidad de casos posibles (22) que presentó el niño en la construcción del espacio muestral, siendo la mitad de ellos favorables y el resto desfavorables al suceso "ganar María". 


\begin{tabular}{|c|c|c|c|c|}
\hline Correcto 1, ruletas & Correcto 2, ruletas & Correcto 3, ruletas & Correcto 4, urnas & Correcto 5, urnas \\
\hline $\begin{array}{c}\text { (C) María y Esteban } \\
\text { tienen posibilidad } \\
\text { de ganar }\end{array}$ & $\begin{array}{c}\text { (C) María y Esteban } \\
\text { tienen posibilidad } \\
\text { de ganar }\end{array}$ & $\begin{array}{c}\text { (C) María y Esteban } \\
\text { tienen posibilidad } \\
\text { de ganar }\end{array}$ & $\begin{array}{c}\text { (C) María y Esteban } \\
\text { tienen posibilidad } \\
\text { de ganar }\end{array}$ & $\begin{array}{c}\text { (C) María y Esteban } \\
\text { tienen posibilidad } \\
\text { de ganar }\end{array}$ \\
\hline
\end{tabular}

Figura 5. Espacios muestrales construidos para un suceso equiprobable.

Hemos encontrado también algunas respuestas incorrectas en este apartado. Por ejemplo, algunos estudiantes utilizan un número mayor de casos favorables, y por tanto mostrarían el sesgo de equiprobabilidad (Lecoutre y Durand, 1988), al considerar equiprobable cualquier resultado de un experimento aleatorio, independientemente del número de casos favorables y posibles. Se muestran ejemplos en la figura 6, el primero de ellos presenta tres casos favorables y solo uno desfavorable, al igual que el segundo. Es posible que el estudiante solo considere sucesos no elementales, sin tener en cuenta su probabilidad, es decir, solo considere que es equiprobable si hay fichas blancas y negras, independientemente de la cantidad. Este resultado concuerda con lo expresado por Francisco y Maher (2005) y Nilsson (2007) quienes señalan que algunos estudiantes que pueden listar todos los sucesos de un espacio muestral tienen dificultad en clasificar los casos en favorables y en desfavorables.

\begin{tabular}{|l|l|}
\hline Incorrecto, ruletas & Incorrecto, urnas \\
\hline
\end{tabular}

Figura 6. Espacios muestrales incorrectos construidos para un suceso equiprobable.

Espacio muestral que corresponde a un suceso imposible. Finalmente, la última tarea consiste en determinar un espacio muestral que haga imposible a María ganar el juego. Algunos ejemplos correctos, uno en contexto de ruletas y otro 
de urnas se presentan en la figura 7; en los dos ejemplos mostrados el estudiante solo incluye casos desfavorables.

\begin{tabular}{|c|c|}
\hline Correcto, ruletas & Correcto, urnas \\
\hline & Mo \\
\hline $\begin{array}{c}\text { (D) Es imposible que } \\
\text { María gane }\end{array}$ & (D) Es imposible que \\
\hline
\end{tabular}

Figura 7. Espacios muestrales construidos para un suceso imposible

Se presentan en la figura 8, ejemplos incorrectos donde se evidencia una confusión entre muy poco probable e imposible, ya citada por Cañizares (1997), Green (1983) y Hernández-Salmerón et al. (2017). Es decir, al considerar el suceso imposible, los estudiantes incluyen algunos casos favorables, incluso en el ejemplo incorrecto 1 de ruletas, aunque proponen más casos favorables que desfavorables. Pensamos que estos estudiantes (una minoría) están atribuyendo a la palabra imposible el sentido de mala suerte, es decir, aunque se tenga bastante probabilidad, todavía se ve el hecho de ganar como algo muy difícil de que ocurra. Cañizares (1997) encontró este tipo de creencias en sus entrevistas a estudiantes sobre la idea de juego equitativo. Además, en el ejemplo incorrecto 4 se muestra como el estudiante confunde poco probable con imposible, ya que mantiene un caso favorable en el espacio muestral.

\begin{tabular}{|c|c|c|c|}
\hline Incorrecto1, ruletas & Incorrecto 2, ruletas & Incorrecto 3, urnas & Incorrecto 4, urnas \\
\hline $\begin{array}{c}\text { (D) Es imposible que } \\
\text { María gane }\end{array}$ & $\begin{array}{c}\text { (D) Es imposible que } \\
\text { María gane }\end{array}$ & $\begin{array}{c}\text { (D) Es imposible que } \\
\text { María gane }\end{array}$ & $\begin{array}{c}\text { (D) Es imposible que } \\
\text { María gane }\end{array}$ \\
\hline
\end{tabular}

Figura 8. Espacios muestrales construidos para un suceso imposible. 
A continuación, se analizan en primer lugar, las frecuencias de respuestas correspondiente a espacios muestrales construidos en contexto de ruletas, seguidamente en contexto de urna y finalmente se comparan los dos contextos. En cada apartado el análisis se realiza globalmente y comparando los resultados de las dos escuelas.

\section{ESPACIOS MUESTRALES CONSTRUIDOS EN CONTEXTO DE RULETAS}

Encontramos diferentes tipos de respuesta en cada apartado del primer ítem. En general, las respuestas han sido variadas, puesto que, para cada tipo de suceso pedido se han construido espacios muestrales asociados a sucesos seguros, posibles, equiprobables, etc. Esto indica una diversidad de intuiciones sobre las ideas de espacio muestral y los tipos de sucesos solicitados. En la tabla 1 presentamos la frecuencia y porcentaje de estudiantes que construyen varios tipos de espacio muestral (correspondiente al suceso María gana seguro, muy probable, equiprobable, poco probable e imposible, en función de lo pedido en cada apartado (seguro, posible, equiprobable e imposible). Se ha marcado en negrita las frecuencias y porcentajes que corresponden a las respuestas correctas.

Tabla 1. Frecuencia y porcentaje de estudiantes en la muestra global, según el espacio muestral construido en cada apartado del ítem 1 (contexto de ruletas).

\begin{tabular}{|c|c|c|c|c|c|c|c|c|}
\hline \multirow{3}{*}{ Espacio muestral construido } & \multicolumn{8}{|c|}{ Apartado del ítem y suceso pedido } \\
\hline & \multicolumn{2}{|c|}{ 1. Seguro } & \multicolumn{2}{|c|}{ 2. Posible } & \multicolumn{2}{|c|}{ 3. Equiprobable } & \multicolumn{2}{|c|}{ 4. Imposible } \\
\hline & $n$ & $\%$ & $n$ & $\%$ & $n$ & $\%$ & $n$ & $\%$ \\
\hline Suceso seguro & 19 & 34,5 & & & & & 1 & 1,8 \\
\hline Suceso muy probable & 23 & 41,8 & 20 & 36,4 & & & 10 & 18,2 \\
\hline Suceso equiprobable & 6 & 11,0 & 27 & 49,1 & 50 & 90,9 & 9 & 16,4 \\
\hline Suceso poco probable & 5 & 9,1 & 6 & 10,9 & 3 & 5,5 & 19 & 34,5 \\
\hline Suceso imposible & & & & & & & 14 & 25,5 \\
\hline No construye & 2 & 3,6 & 2 & 3,6 & 2 & 3,6 & 2 & 3,6 \\
\hline
\end{tabular}

Son muy pocos los estudiantes que no completaron la tarea, lo cual indica el interés con que colaboraron a nuestro trabajo. Los mejores resultados se 
obtienen al construir un espacio muestral donde el suceso "María gana", sea un suceso posible, correctamente contestado por todos los estudiantes que participaron en este apartado (96,4\%). Esto indica una buena intuición de la idea de suceso posible, lo que se debe sin duda a que en este apartado se pueden admitir como correctas muchas respuestas (muy probable, equiprobable, poco probable). También, se obtuvo buen rendimiento al construir el espacio muestral asociado al suceso equiprobable, cuya construcción fue correcta para una amplia mayoría.

Observamos la mayor dificultad en el suceso seguro, interpretado con alta frecuencia como muy probable, y también en el suceso imposible, considerado por una proporción importante como poco probable; en estos dos tipos de sucesos aparecen otras interpretaciones en porcentajes importantes de estudiantes, en concreto incluso se observa confusión entre suceso muy y poco probable.

En la tabla 2 se comparan los resultados del ítem 1 separados por escuela, donde se repiten en cada grupo los resultados observados en la tabla 1, salvo una pequeña variación en el número de no respuestas por escuela. Es decir, en el contexto de ruletas donde se aprecian pocas diferencias porcentuales en los espacios muestrales construidos de acuerdo al suceso solicitado. Cabe señalar que los estudiantes de la escuela pública tuvieron todas las respuestas correctas en el suceso equiprobable "María y Esteban tienen igual posibilidad de ganar"; y los estudiantes de la escuela privada tuvieron un rendimiento levemente mejor $(31,0 \%)$ respecto a los de la escuela pública $(19,2 \%)$ al responder correctamente el suceso imposible.

Tabla 2. Porcentaje de respuestas en cada apartado del ítem 1 (ruletas) por escuela.

\begin{tabular}{lrrrrrrrr}
\hline \multirow{2}{*}{$\begin{array}{l}\text { Espacio muestral } \\
\text { construido }\end{array}$} & \multicolumn{3}{c}{ Suceso seguro } & \multicolumn{2}{c}{ Posible } & \multicolumn{2}{c}{ Equiprobable } & \multicolumn{2}{c}{ Imposible } \\
\cline { 2 - 9 } & Priv. & Púb. & Priv. & Púb. & Priv. & Púb. & Priv. & Púb. \\
\hline Suceso seguro & 34,5 & 34,6 & & & & & & 3,8 \\
Suceso muy probable & 41,4 & 42,3 & 31,0 & 42,3 & & & 10,3 & 26,9 \\
Suceso equiprobable & 6,9 & 15,4 & 51,7 & 46,2 & 82,8 & 100,0 & 13,8 & 19,2 \\
Suceso poco probable & 10,3 & 7,7 & 10,3 & 11,5 & 10,3 & & 37,9 & 30,8 \\
Suceso imposible & & & & & & & 31,0 & 19,2 \\
No construye & 6,9 & & 6,9 & & 6,9 & & 6,9 & \\
\hline
\end{tabular}




\section{ESPACIOS MUESTRALES CONSTRUIDOS EN CONTEXTO DE URNAS}

En la tabla 3 presentamos la frecuencia y porcentaje de estudiantes que construyen varios tipos de espacio muestral en el ítem 2, contexto de urnas en cada apartado (suceso "gana María" seguro, posible, equiprobable e imposible). Se ha marcado en negrita las frecuencias y porcentajes que corresponden a las respuestas correctas.

Tabla 3. Frecuencia y porcentaje de estudiantes en la muestra global, según el espacio muestral construido en cada apartado del ítem 2 (contexto de urnas).

\begin{tabular}{lrrrrrrrr}
\hline \multicolumn{1}{c}{ Apartado del item y suceso pedido } \\
\hline $\begin{array}{l}\text { Espacio muestral } \\
\text { construido }\end{array}$ & 1. Seguro & 2. Posible & 3. Equiprobable & 4. Imposible \\
\cline { 2 - 11 } & \multicolumn{1}{c}{$\%$} & \multicolumn{1}{c}{$n$} & $\%$ & $n$ & $\%$ & $n$ & $\%$ \\
\hline Suceso seguro & 16 & 29,1 & & & & & & \\
Suceso muy probable & 19 & 34,6 & 16 & 29,1 & 2 & 3,6 & 6 & 10,9 \\
Suceso equiprobable & 1 & 1,8 & 7 & 12,8 & 39 & 70,9 & 2 & 3,6 \\
Suceso posible & 17 & 30,9 & 28 & 50,9 & 13 & 23,7 & 15 & 27,3 \\
Suceso poco probable & 1 & 1,8 & 2 & 3,6 & & & 17 & 30,9 \\
Suceso imposible & & & & & & & 13 & 23,7 \\
No construye & 1 & 1,8 & 2 & 3,6 & 1 & 1,8 & 2 & 3,6 \\
\hline
\end{tabular}

Aquí también es importante resaltar el interés por parte de todos los estudiantes de la muestra para completar el cuestionario ya que son muy pocas las no respuestas. Al igual que el ítem de contexto ruletas, donde hubo mejores resultados fue al construir el espacio muestral correspondiente al suceso posible, correctamente respondido por toda la muestra que realizó el apartado (96,4\%). También hubo buen rendimiento en el suceso equiprobable, donde 7 de cada 10 estudiantes respondieron correctamente. Al igual que el ítem de contexto de ruletas, observamos la mayor dificultad en el suceso seguro, interpretado con alta frecuencia como muy probable o posible, y el suceso imposible, generalmente considerado como poco probable o posible; en estos dos tipos de sucesos aparecen también otras interpretaciones. 
En la tabla 4 se comparan los resultados por escuela en el contexto de urnas, donde se aprecian pocas diferencias porcentuales en los espacios muestrales construidos de acuerdo al suceso solicitado. Los estudiantes de la escuela privada tuvieron un rendimiento levemente mejor $(27,6 \%)$ respecto a los de la escuela pública (19,2\%) en responder correctamente el suceso imposible, y también, un rendimiento mayor aproximadamente del 10\% de respuestas correctas en el de suceso equiprobable.

Tabla 4. Porcentaje de respuestas en cada apartado del ítem 2 (urnas) por escuela.

\begin{tabular}{lrrrrrrrr}
\hline & \multicolumn{8}{c}{ Tipo de suceso pedido } \\
\hline $\begin{array}{l}\text { Espacio muestral } \\
\text { construido }\end{array}$ & \multicolumn{2}{c}{ Seguro } & \multicolumn{2}{c}{ Posible } & Equiprobable & \multicolumn{2}{c}{ Imposible } \\
\cline { 2 - 10 } & Priv. & Púb. & Priv. & Púb. & Priv. & Púb. & Priv. & Púb. \\
\hline Suceso seguro & 27,6 & 30,8 & & & & & & \\
Suceso muy probable & 27,6 & 42,3 & 31,0 & 26,9 & 6,9 & & 6,9 & 15,4 \\
Suceso equiprobable & & 3,8 & 6,9 & 19,2 & 75,9 & 65,4 & & 7,7 \\
Suceso posible & 37,9 & 23,1 & 55,2 & 46,2 & 13,8 & 34,6 & 27,6 & 26,9 \\
Suceso poco probable & 3,4 & & 3,4 & 3,8 & & & 31,0 & 30,8 \\
Suceso imposible & & & & & & & 27,6 & 19,2 \\
No construye & 3,4 & & 3,4 & 3,8 & 3,4 & & 6,9 & 3,7 \\
\hline
\end{tabular}

\section{COMPARACIÓN DE RESULTADOS POR CONTEXTO}

En la tabla 5 se comparan las respuestas de los estudiantes en porcentajes de acuerdo al contexto del ítem en cada uno de los apartados, señalando en negrita las respuestas correctas. 
Tabla 5. Porcentaje espacios muestrales construidos según suceso pedido por contexto.

\begin{tabular}{|c|c|c|c|c|c|c|c|c|}
\hline \multirow{3}{*}{$\begin{array}{l}\text { Espacio muestral } \\
\text { construido }\end{array}$} & \multicolumn{8}{|c|}{ Apartado del ítem y suceso pedido } \\
\hline & \multicolumn{2}{|c|}{ 1. Seguro } & \multicolumn{2}{|c|}{ 2. Posible } & \multicolumn{2}{|c|}{ 3. Equiprobable } & \multicolumn{2}{|c|}{ 4. Imposible } \\
\hline & Ruleta & Urna & Ruleta & Urna & Ruleta & Urna & Ruleta & Urna \\
\hline Suceso seguro & 34,5 & 29,1 & & & & & 1,8 & \\
\hline Suceso muy probable & 41,8 & 34,6 & 36,4 & 29,1 & & 3,6 & 18,2 & 10,9 \\
\hline Suceso equiprobable & 11,0 & 1,8 & 49,1 & 12,8 & 90,9 & 70,9 & 16,4 & 3,6 \\
\hline Suceso posible & & 30,9 & & 50,9 & & 23,7 & & 27,3 \\
\hline Suceso poco probable & 9,1 & 1,8 & 10,9 & 3,6 & 5,5 & & 34,5 & 30,9 \\
\hline Imposible & & & & & & & 25,0 & 23,7 \\
\hline No construye & 3,6 & 1,8 & 3,6 & 3,6 & 3,6 & 1,8 & 3,6 & 3,6 \\
\hline
\end{tabular}

Globalmente y en diferente grado, se aprecia una menor dificultad en la construcción de espacios muestrales en el contexto ruletas, siendo la mayor diferencia en el suceso equiprobable, donde $20 \%$ más de los estudiantes lograron responder correctamente este apartado, seguido por el de los sucesos seguro e imposible donde hay una diferencia del 5,4\% y 1,8\% respectivamente en las respuestas correctas. El suceso posible no presenta diferencias porcentuales; sin embargo, sí hay mayor diversidad en las respuestas en el contexto urnas, debido a que es una tarea que presenta menos restricciones.

En la tabla 6, se presenta la distribución del número de apartados correctos en cada uno de los dos contextos, donde el máximo posible serían 4 respuestas correctas.

Tabla 6. Frecuencia y porcentaje de número de respuestas correctas en los dos contextos.

\begin{tabular}{crrrrrc}
\hline Respuestas correctas & \multicolumn{3}{c}{ Ítem 1. Ruletas } & \multicolumn{3}{c}{ Ítem 2. Urnas } \\
\hline & $n$ & $\%$ & $\begin{array}{c}\text { Porcentaje } \\
\text { acumulado }\end{array}$ & $n$ & $\%$ & $\begin{array}{c}\text { Porcentaje } \\
\text { acumulado }\end{array}$ \\
& 3 & 5,5 & 5,5 & 2 & 3,6 & 3,6 \\
1 & 26 & 47,2 & 52,7 & 23 & 41,8 & 45,4 \\
2 & 10 & 18,2 & 70,9 & 16 & 29,1 & 74,5 \\
3 & 9 & 16,4 & 87,3 & 9 & 16,4 & 90,9 \\
4 & 7 & 12,7 & 100,0 & 5 & 9,1 & 100,0 \\
\hline
\end{tabular}


Al comparar el número de respuestas correctas en cada ítem, observamos que siete estudiantes en el primer ítem y cinco en el segundo logran responder correctamente los cuatro apartados de cada ítem. Por otro lado, se dan porcentajes muy similares en cada contexto respecto al número de tareas resueltas correctamente. El porcentaje de estudiantes que contesta adecuadamente a dos preguntas o menos en ambos contextos ronda el 70\%; sin embargo, hay que señalar que en el contexto urnas el 54,6\% de los estudiantes respondieron 2 o más apartados correctamente, a diferencia del contexto de ruletas donde esto ocurrió en el 47,3\% de los estudiantes.
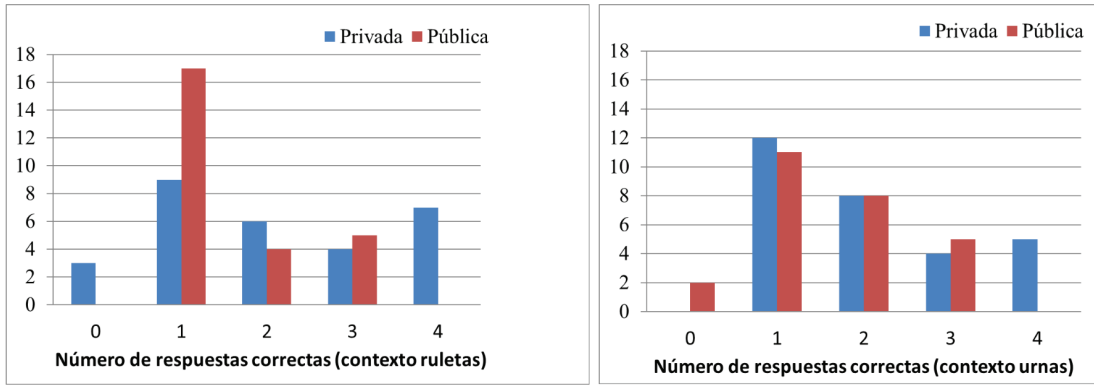

Figura 9. Distribución del número de respuestas correctas en cada contexto según tipo de escuela.

En la figura 9 se comparan el número de respuestas correctas en cada contexto según tipo de escuela. En general, los estudiantes de la escuela privada tuvieron mejores rendimientos en ambos contextos respecto a los de la escuela pública. En el contexto de ruletas se observa que 65,4\% de los estudiantes de la escuela pública solo tuvieron un apartado correcto, a diferencia de los de la escuela privada donde 58,6\% tuvieron más de uno. Respecto al contexto de urnas, todos los estudiantes de la escuela privada al menos lograron un apartado correcto, a diferencia de los de escuela pública. Además, mientras ningún niño de la escuela pública obtuvo los cuatro apartados correctos en los dos ítems, en la escuela privada $24,1 \%$ de los estudiantes lo logró en contexto de ruletas y $17,2 \%$ en el de urnas. 


\section{DISCUSIÓN E IMPLICACIONES PARA EL AULA}

Como se indicó en la introducción, la habilidad general que se pretende lograr al final del ciclo educativo es identificar distintos sucesos (más probables, menos probables o igualmente probables) de acuerdo con el número de resultados simples de cada uno de estos. En las tareas propuestas se debe construir un espacio muestral a partir de ciertas condiciones iniciales, lo que plantea un desafío para el estudiante al no ser una tarea usual.

Los resultados del estudio muestran una intuición razonable de la idea de espacio muestral en la muestra de estudiantes que han participado, tanto en contexto de urnas, como en contexto de ruletas. Estos han sido capaces, en su mayoría, de construir un espacio muestral adecuado cuando en el enunciado de las tareas se solicitó un suceso posible y un suceso equiprobable. Este hecho señala que los estudiantes ya han cimentado un paso importante en su avance en el estudio de la probabilidad, según Bryant y Nunes (2012).

Se ha podido constatar, sin embargo, al igual que en las investigaciones de Cañizares (1997), Green (1983) y Hernández-Salmerón et al. (2017), que algunos estudiantes confunden el suceso seguro con el suceso muy probable, y el suceso imposible con el suceso muy poco probable. Pero, además, hemos encontrado otras intuiciones erróneas, como considerar equiprobable un suceso con más casos favorables que desfavorables (o viceversa), considerar seguro un suceso poco probable o considerar seguro un suceso improbable. Atendiendo a las indicaciones de Fischbein (1975), hay que considerar con atención las intuiciones primarias sobre el significado de los tipos de sucesos que los estudiantes traen a la clase, pues las intuiciones incorrectas son muy difíciles de cambiar.

Estos hallazgos indican también la necesidad de prestar mayor cuidado al lenguaje de probabilidad que se utiliza en la clase, puesto que los estudiantes no solo le asocian significados del contexto cotidiano que no corresponden al matemático, sino también significados muy diferentes, tanto a lo cotidiano como a lo matemático. Nos parece que el lenguaje del azar es sencillo para el niño, pero a veces no se atiende el hecho de que detrás de cada término hay conceptos asociados cuya intuición primero y comprensión posterior requiere de tiempo y esfuerzo, si queremos que el niño progrese adecuadamente en su estudio posterior de la probabilidad.

Pero el lenguaje influye en el conocimiento informal de probabilidad de los estudiantes, con el que llegan a la escuela e interpretan las tareas de probabilidad que les planteamos (Amir y Williams, 1999). Molnar (2018) recuerda que 
las ambigüedades del lenguaje en probabilidad pueden llevar a la formación de intuiciones erróneas, y que el profesor debe estar atento al significado que los estudiantes dan a los términos probabilísticos.

Será, entonces, importante dedicar un tiempo a que los estudiantes refuercen este lenguaje y realicen tareas como las propuestas en que ellos mismos deben explicitar el espacio muestral del experimento. Nunes, Bryant, Evans, Gottardis y Terlektsi (2014) indican que es posible apoyar la idea de espacio muestral a partir de los 10 años, cuando se comienzan a desarrollar los esquemas conceptuales de clasificación, conjunción o razón, que utilizan en otros temas matemáticos.

Igualmente tendrán gran importancia los generadores de sucesos aleatorios, tanto manipulativos (dados, ruletas, fichas en urnas, etc.) como los representados gráficamente que se hayan utilizado en las situaciones propuestas a los estudiantes, pues no todos tienen las mismas propiedades (Gandhi, 2018). Aunque aparentemente no ha habido diferencias notables en el número de niños que ha dado respuesta correcta para cada apartado en los dos contextos considerados, hemos notado que el número total de respuestas correctas es mayor en el contexto de ruletas, que al haberse dividido solo en cuartos ha facilitado la comparación de probabilidades a los estudiantes. Recomendamos, por tanto, el uso de una variedad de tareas y dispositivos manipulativos en la enseñanza de la probabilidad a estos estudiantes.

\section{AGRADECIMIENTO}

Proyecto PID2019-105601GB-I00/ AEI / 10.13039/501100011033 y Grupo FQM126 (Junta de Andalucía).

\section{REFERENCIAS}

Alpízar, M., Barrantes, J., Bolaños, H., Céspedes, M., Delgado, E., Freer, D., Padilla, E., y Víquez, M. (2012). Aspectos relevantes sobre la formación docente en I y II ciclos en los temas probabilidad y estadística. Educare, 16(2), 113-129.

Alpízar, M., Chavarría, L. y Oviedo, K. (2015). Percepción de un grupo de docentes de I y Il ciclo de educación general básica de escuelas públicas de Heredia sobre los temas 
de estadística y probabilidad. Actualidades Investigativas en Educación, 15(1), 1-23. https://dx.doi.org/10.15517/aie.v15i1.17728

Amir, G. S., y Williams, J. S. (1999). Cultural influences on children's probabilistic thinking. The Journal of Mathematical Behavior, 18(1), 85-107. https://doi.org/10.1016/S07323123(99)00018-8

Australian Curriculum, Assessment and Reporting Authority (ACARA) (2013). The Australian curriculum: Mathematics. Autor.

Bryant, P. y Nunes, T. (2012). Children's understanding of probability: A literature review. Nuffield Foundation

Borovcnik, M. y Bentz, H. J. (1991). Empirical research in understanding probability. En M. Borovnick y R. Kapadia (Eds.), Chance encounters: Probability in education (pp. 73-105). Springer.

Cañizares, M. J. (1997). Influencia del razonamiento proporcional y combinatorio y de creencias subjetivas en las intuiciones probabilísticas primarias (Tesis doctoral no publicada). Universidad de Granada.

Common Core State Standards Initiative, CCSSI (2010). Common Core State Standards for Mathematics. Washington, DC: National Governors Association Center for Best Practices and the Council of Chief State School Officers. www.corestandards.org/assets/ CCSSI_Math\%20Standards.pdf.

Ernest, P. (1984). Introducing the concept of probability. Mathematics Teacher, 77, 524- 525. Fischbein, E. (1975). The intuitive sources of probability thinking in children. Reidel.

Fischbein, E. (1987). Intuition in science and mathematics. Reidel

Fischbein, E. y Gazit, A. (1984). Does the teaching of probability improve probabilistic intuitions? Educational Studies in Mathematics, 15(1), 1-24.

Francisco, J. M. y Maher, C. A. (2005). Conditions for promoting reasoning in problem solving: Insights from a longitudinal study. Journal of Mathematical Behavior, 24(2-3), 361-372. Franklin, C., Kader, G., Mewborn, D., Moreno, J., Peck, R., Perry, M, y Scheaffer, R. (2007). Guidelines for assessment and instruction in statistics education (GAISE) report: A Pre-K-12 curriculum framework. American Statistical Association. http://www.amstat. org/Education/gaise/

Gandhi, H. (2018). Understanding children's meanings of randomness in relation to random generators. En C. Batanero y E. Chernoff (Eds.). Teaching and Learning Stochastics (pp. 181-200). Springer. https://doi.org/10.1007/978-3-319-72871-1_11

Godino, J. D., Batanero, C. y Font, V. (2007). The onto-semiotic approach to research in mathematics education. ZDM. The International Journal on Mathematics Education, 39(1-2), 127-135. https://doi.org/10.1007/s11858-006-0004-1. 
Gómez, E., Ortiz, J. J., Batanero, C. y Contreras, J. M. (2013). El lenguaje de probabilidad en los libros de texto de Educación Primaria. Unión 35, 75-91.

Green, D. R. (1983). School pupils' probability concepts. Teaching Statistics, 5(2), 34-42.

Hernández, E. (2015). El lenguaje del azar en alumnos de educación secundaria obligatoria. (Tesis de maestría no publicada). Universidad de Granada.

Hernández-Salmerón, E., López-Martín, M. M. y Batanero, C. (2017). Estudio exploratorio sobre el lenguaje del azar en educación secundaria obligatoria. En J. M. Muñoz, A. Arnal-Bailera, P. Beltrán-Pellicer, M. L. Callejo y J. Carrillo (Eds.), Investigación en Educación Matemática XXI (pp. 305-314). Universidad de Zaragoza.

Hernández-Solís, L., Batanero, C., Gea, M. y Álvarez-Arroyo, R. (2021). Comparing probabilities in urns: A study with primary school students. Uniciencia, 35(2), 1-18. https:// doi.org/10.15359/ru.35-2.9

Horvath, J. K. y Lehrer, R. (1998). A model-based perspective on the development of children's understanding of chance and uncertainty. En S. Lajoie (Ed.), Reflections on statistics: learning, teaching, and assessment in grades K-12 (pp. 121-148 ). Routledge. Jones, G., Langrall, C., y Mooney, E. (2007). Research in probability: responding to classroom realities. En F. Lester (Ed.), Second handbook of research on mathematics teaching and learning (vol. 2, pp. 909-955). Information Age Publishing y NCTM.

Keren, G. (1984). On the importance of identifying the correct 'problem space'. Cognition, $16,121-128$.

Langrall, C. y Mooney, E. (2005). Characteristics of elementary school students' probabilistic reasoning. En G. Jones (Ed.), Exploring probability in school (pp. 95-119). Springer.

Lecoutre, M. P. y Durand, J. L. (1988). Judgements probabilistes et modèles cognitifs: étude d'une situation aleatoire. Educational Studies in Mathematics, 19, 357-368.

Maury, S. (1984). La quantification des probabilites: analyse des argumentes utilises par les eleves de classe de seconde. Recherches en Didactique des Mathematiques, 5(2), $187-215$.

Ministerio de Educación, Cultura y Deporte, MECD (2014). Real Decreto 126/2014, de 28 de febrero, por el que se establece el currículo básico de la Educación Primaria. Autor.

Ministerio de Educación Pública (MEP). (2012). Programas de Estudio de Matemáticas. I, II y III Ciclos de la Educación General Básica y Ciclo Diversificado. Autor.

Molnar, A. (2018). Language and lexical ambiguity in the probability register. En C. Batanero y A. Chernoff (Eds.), Teaching and learning stochastics (pp. 23-37). Springer. https://doi.org/10.1007/978-3-319-72871-1_2.

Nacarato, A. M. y Grando, R. C. (2014). The role of language in building probabilistic thinking. Statistics Education Research Journal, 13(2), 93. 
National Council of Teachers of Mathematics (NCTM) (2000). Principles and standards for school mathematics. NCTM.

Nilsson, P. (2007). Different ways in which students handle chance encounters in the explorativesetting of a dice game. Educational Studies in Mathematics, 66(3), 293315. https://doi.org/10.1007/s10649-006-9062-0.

Nunes, T., Bryant, P., Evans, D., Gottardis, L. y Terlektsi, M. E. (2014). The cognitive demands of understanding the sample space. ZDM Mathematics Education, 46(3), 437-448.

Ortiz, J. J., Albanese, V. y Serrano, L. (2016). El lenguaje de la estadística y probabilidad en libros de texto de Educación Secundaria Obligatoria. En J. A. Macías, A. Jiménez, J. L. González, M. T. Sánchez, P. Hernández, C. Fernández, F. J. Ruiz, T. Fernández y A. Berciano (Eds.), Investigación en Educación Matemática XX (pp. 397-406). SEIEM.

Pratt, D. y Kazak, S. (2018). Research on uncertainty. In International handbook of research in statistics education (pp. 193-227). Springer. https://doi.org/10.1007/978-3-31966195-7_6

Schleppegrell, M. (2007). The linguistic challenges of mathematics teaching and learning: A research review. Reading and Writing Quarterly, 23, 139-159. https://doi. org/10.1080/10573560601158461

Vásquez. C. y Alsina, Á. (2015). El conocimiento del profesorado para enseñar probabilidad: Un análisis global desde el modelo del conocimiento didáctico-matemático. Avances de Investigación en Educación Matemática, 7, 27-48. https://doi.org/10.35763/aiem.vli7.104.

Vásquez, C. y Alsina, Á. (2017). Aproximación al conocimiento común del contenido para enseñar probabilidad desde el modelo del Conocimiento Didáctico-matemático. Educación matemática, 29(3), 79-108.

Vygotski, L. S. (2012). Thought and language. MIT press.

Watson, J. (2005). The probabilistic reasoning of middle school students. En G. A. Jones (Ed.), Exploring probability in school (pp. 145-169). Springer.

Watson, J. M. y Moritz, J. B. (2010). The development of comprehension of chance language: Evaluation and interpretation. School Science and Mathematics, 103(2), 65-80. https://doi.org/10.1111/j.1949-8594.2003.tb18222.x

MARÍA M. GEA

Dirección: Facultad de Ciencias de la Educación, Campus de Cartuja, 18071, Granada, España 